\title{
INVESTIGACIÓN/RESEARCH
}

Recibido: 04/06/2014 Aceptado: 01/09/2014 $\quad$ Publicado: 15/11/2014

\section{EL JOVEN MODELO: PRINCIPIOS DE URBANIDAD PARA EL USO DE LA JUVENTUD DE PÍO DEL CASTILLO}

Geraldine Patricia Chacón Villarroel. Universidad Metropolitana. Caracas. (Venezuela) geraldine.chacon@sciencespo.fr

Del Valle Mejías María Elena. Universidad Metropolitana. Caracas (Venezuela) manedelvalle@gmail.com

RESUMEN: Los manuales de urbanidad reflejan la fisionomía cultural de un panorama local. En América Latina fueron varios los manuales que se destacaron como las biblias para modelar a la juventud en cuanto a normas de civilidad y ciudadanía. Este trabajo se enfoca en evaluar el manual del colombiano Pío del Castillo, uno de los textos menos trabajados en el estudio de manuales y que resulta un complejo compendio de formas civiles que, a través de la clara instrucción, tanto moral como física, dibuja lo que en el contexto del siglo XIX se recupera como el joven modelo. Se realiza un bosquejo de múltiples autores que evalúan el tema de la civilidad y el rol de los manuales en la formación de la misma; su relación con la civilidad europea, la radicalidad o severidad de la instrucción y el rol del Estado como formador. Se descubre a través del análisis crítico de la obra que la configuración de un ciudadano no se limita a maneras de cortesía sino a toda una red que dibujar el comportamiento y la personalidad ideal para el siglo.

PALABRAS CLAVE: Manual de Urbanidad - Colombia - Juventud - Pío Del Castillo Siglo XIX

\section{THE YOUNG MODEL: PRINCIPLES OF CIVILITY FOR THE USE OF YOUTH}


ABSTRACT: Etiquette manuals reflect the culture physiognomy of a local scene. In Latin America there were several manuals that were highlighted as bibles for youth modeling in standards of civility and citizenship. This work focuses on evaluating the Colombian manual by Pío del Castillo; one of the least worked on the study of manuals, and that it presents a complex compendium of urbanity norms that, through clear instruction, both moral and physical, it draws what, in the nineteenth century context, is recovered as the young model. Multiple authors' views are outlined, evaluating the issue of civility and the role of textbooks and manuals in the formation process of it; its relationship with European civility, the radical or severity of instruction and the State's role as a trainer. It is discovered through the critical analysis of the manual that the configuration of a citizen is not limited to polite ways but to a whole network that draws the ideal behavior for the century.

KEY WORDS: Etiquette manual - Colombia - Principles of Civility - Pío Del Castillo Nineteenth Century

\section{ÍNDICE}

1. Introducción: Principios de Urbanidad para el Uso de la Juventud de Pío del Castillo (Colombia)

1. 1 Análisis Crítico y Contraste con múltiples Autores

2. Bibliografía

En palabras de Luis Alarcón Meneses, los manuales de educación cívica "representaban en aquel tiempo el camino ideal para alcanzar el progreso y la civilización" (Alarcón, 2012) y efectivamente el manual que aquí trabajamos busca modelar al joven, de forma que éste se ajuste a la sociedad como el mejor ciudadano que pueda ser. Los principios de Urbanidad para el Uso de la Juventud recopilan entonces, de forma bastante didáctica para la época, una cantidad de valores que buscan instruir al joven en las maneras más civiles y educadas que el autor puede concebir.

Sin embargo, Pío Castillo en este manual desarrolla aspectos tanto espirituales y morales, como físicos; alcanzando desde los deberes con Dios hasta las correctas maneras de aseo personal y presencia. Esto ilustra de cierta forma que la construcción de un joven modelo, de un ciudadano modelo, en este contexto, se escapa de un solo aspecto de sí mismo, sino que se considera un todo, un conjunto global de maneras y actitudes que involucran comportamientos y pensamientos hasta actuaciones que se 
exponen en el mundo exterior. El manual desarrolla entonces, como idea introductoria, el planteamiento de la ciudadanía y urbanidad como una ciencia fundamental para la convivencia en sociedad. (Castillo, 1841) Madiedo, contemporáneo del siglo, sostiene una posición similar, donde se destaca como defensor de la civilidad, pues ésta, como formación moral y social resulta la manera de evolución de humanidad. (Madiedo, 1863) En definitiva, para el profesor Pío Castillo la vida común de los ciudadanos no puede ser posible si éstos no siguen severamente esta serie normativa que lo que busca en esencia, es regular la vida moral y presencial de los jóvenes. Es toda una compleja red de normas sociales y personales. Esta red sin embargo, se constituye a sí misma en un entorno de urbanidad, lo que limita su alcance a un determinado círculo social de la comunidad colombiana del siglo XIX; que, como plantea Leslie Bethell, no era el único, pues la población entera de esta sociedad no estaba conformada únicamente por círculos sociales urbanos, sino que el componente campesino constituye un elemento sustancial para la fisionomía poblacional de la Colombia del siglo XIX. (Bethell, 1986)

Esta idea permite ubicar entonces, que esta red compleja de normas sociales no se conforma como un conjunto de principios universales para la sociedad colombiana sino que más bien, ilustran el afán civilizatorio y el ahínco en la fineza de la parte de la población más urbanizada. Aun así, no debe subsumirse en características aisladas el contenido que plantea este texto solo porque se enmarque en un círculo social particular. Como manual de formación tiene la capacidad, en definitiva, de revelar estructuras sociales del ideal de la construcción de la nación a través de la formación de sus ciudadanos, pues resulta innegable que el libro y la instrucción en urbanidad eran valoradas por las élites sociales -que aunque no mayoritarias, fundamentales- $y$ por ende deben ser consideradas "un referente ideal dentro del discurso sobre la instrucción pública" (Alarcón, 2012).

De esta forma se configura un modelo, un estándar de "correcto" y "apropiado" que busca determinar el desarrollo de la sociedad, donde al parecer, una nación incipiente busca equipararse a la bien formada cultura europea. Vale la pena acotar a su vez lo que Alarcón plantea, extrayendo de informes de la Gobernación de la Provincia de Barranquilla (1869) que "En algunas escuelas, incluso de capitales de provincia, no se prestaba atención a la enseñanza de la historia nacional y a la instrucción cívica. Los manuales escolares eran escasos." (Alarcón, 2012) A partir de esto, el manual de Pío Castillo podría tomarse como prematuro (data de 1841) en cuanto su objeto pudo estar al margen del objeto de las materias más difundidas en el sistema escolar para la época; empero, no puede negarse la importancia o valoración que se le diera a la instrucción sobre urbanidad.

Habiendo realizado estas consideraciones previas sobre la relevancia del manual de urbanidad y su sentido como modelo de instrucción, valorado y promovido por las élites para la construcción de un determinado ciudadano; resulta apropiado abordar el propio texto de Pío del Castillo y evaluar de qué forma configura éste el joven modelo colombiano para el siglo XIX. 
Jaime Jaramillo Uribe, autor de gran relevancia para la historiografía colombiana, dibuja la estructura educativa en su obra "El pensamiento colombiano en el siglo XIX" (1964) donde, si bien desarrolla con profundidad el pensamiento independentista de los héroes nacionales, destaca algo interesante: El colombiano de este siglo "mira con buenos ojos las formas de vida francesas y anglosajonas" (Jaramillo, 1964) lo que cristaliza la tesis en la que planteamos que la urbanidad pensada por Pío Castillo, las buenas maneras con las que se pretende educar al niño y al joven, se configuran como estructuras sociales inspiradas por las formas de vida europeas.

En un estudio publicado por la revista de Historia Iberoamericana en el 2007, Claudia Venegas, historiadora de la Universidad Nacional de Colombia asevera que "el país quiso hacer parte de los países civilizados y se propuso importar la modernidad de Europa. Para los gobernantes de la época, difundir los buenos modales fue, entonces, una vía para alcanzar el primer mundo: refinado y glamoroso" (Venegas, 2007). Sin embargo, pareciera que estos modales dejaran de ser formalidades y buenas maneras banales para ser, para toda una carga de educación moral y cívica justa y necesaria.

Manuel María Madiedo, consideraba incluso que ésta educación moral era una responsabilidad del Estado y los intelectuales, frente a su población, la "masa ignorante". (Madiedo, 1863). Evelyn Ahern, en su obra El desarrollo de la Educación en Colombia: 1820 - 1850 plantea que luego del período independentista, lo transmitido por la educación "se trataba de formar ciudadanos para un país que emprendía el largo proceso de construcción nacional" (Ahern, 1947)

Y efectivamente, el manual de Pío Castillo plantea un fuerte contenido de formación moral con miras a la construcción de un hombre de sociedad -valioso para su nacióndonde, desde su primer capítulo, elabora una serie de máximas de comportamiento que ensalzan los deberes con Dios, con los padres y con demás autoridades.

Estos deberes se plantean con una radicalidad que, en principio, podría considerarse propia de la época pero que al realizar un sondeo, tanto sobre otros manuales de urbanidad de la región, como en los esquemas de las instituciones de formación, revela que Pío del Castillo sostiene una severidad un tanto más destacable que otros autores. Por ejemplo, en las Lecciones de Urbanidad de Rufino Cuervo (1836), no se encuentran aseveraciones del calibre de Castillo cuando afirma:

El joven que no ama a sus padres (...) se atrae sobre sí la maldición del Señor, el desprecio de sus semejante i(sic) sobre él recaen cuantas desgracias son consiguientes de tan enorme delito: nunca disfrutará sociego(sic) ni bienes sobre la tierra, preparándose por su mano perdurables tormentos para la eternidad. (Del Castillo, 1841.)

¿Qué busca Pío Del Castillo al presentar argumentos con tal severidad? Pareciera que es una instrucción moral, con un riguroso contenido religioso, que busca de una forma u otra determinar al joven a seguir la pauta de forma inequívoca y sin salida alguna. Se presenta como elemental entonces un carácter implícitamente cristiano católico en el joven. Esta referencia resulta interesante de evaluar, pues refleja que el proceso 
independentista no implicó certeras separaciones de las provincias americanas de la cultura europea, cacaso no es el catolicismo un rasgo primordial heredado de la colonia?

A lo largo de todo el texto, en cuanto a deberes con terceros, el contenido religioso tiene una presencia fuerte. La conclusión es inevitable: La formación moral, aún en este período histórico de la incipiente Colombia, se construye a partir de la formación religiosa, y son los valores cristianos los que plantean la base de toda enseñanza en cuanto a morales se refiere. Esta moralidad, se confundirá a su vez con un sentido un tanto patriótico, adoptando los simbolismos religiosos y la pasionalidad de la moral cristiana a un deber inagotable de servidumbre ante la nación, a la sociedad y este manual particular, a los terceros.

Esta sostenida idea de servidumbre frente a los otros configura lo que parece, tanto de forma como de fondo, un joven esencialmente sumiso, caracterizado más bien por una personalidad guiada a la discreción total y más bien vaciado de cualquier rasgo personal que pudiera interpretarse como altivez pues cualquier actitud que se escape de este margen de civilidad con algún ahínco o pasión inadecuada, se convierte en grosería y según Del Castillo, es esto lo más condenatorio para un joven que pretende vivir en sociedad. Incluso varios capítulos se dedican exclusivamente a plantear máximas o frases de cortesía que el joven debe seguir (memorizar, si se es más apropiado); lo que refleja esta seriedad a la cual el joven se ve sometido; donde mantenerse no sólo dentro de unos lineamientos que lo vacían de altivez sino que debe seguir una línea marcada de conducta. Es reiterado el pensamiento que presenta esta cortesía-sumisión ante los demás. Y sobre esta idea vale la pena posar la mirada con mayor atención.

Pío Del Castillo, así como enuncia con severidad religiosa consecuencias por incumplimiento o descarríos; destaca con ahínco a lo largo de todo el texto la intención fundamental que debe tener el joven: Ser servicial y respetuoso. Desde las actitudes más introvertidas frente a autoridades hasta cortesías con personas de clase inferior, el epicentro de todas las buenas costumbres para Del Castillo se encuentra en la "no molestia al otro", la idea de todo el protocolo se fundamenta en no perturbar el orden social, y ser más bien quien aporte virtud y urbanidad, para así serle útil a la sociedad.

Esto lleva a reflexionar sobre un rasgo característico del manual estudiado, y es la explotación de la idea que no mide al individuo por su clase social o status quo determinado, sino que más bien valora su virtud, urbanidad y buenas maneras, haciendo de la buena educación, un elemento civilizatorio más allá de la estirpe o condición social generalmente estática, y esta idea se afirma en las instrucciones de civismo que brinda el manual; considérese por ejemplo: "Iguales pues, sean las atenciones que usemos con el pobre que con el rico, concediendo solo la diferencia a la virtud i (sic) al mérito de la persona, sea la que fuere su clase o condición." (Del Castillo, 1841)

También se dibuja en las fábulas y versos finales, donde, al extraer mensajes y valoraciones de diversos cuentos cortos, se ilustra que la correcta virtud no se basa en 
características superficiales sino en la utilidad que como individuo puede brindar el joven a través de su formación moral. Se sostiene una premisa donde la virtud es valorada de forma natural por la sociedad que las recibe, y es bajo esta perspectiva que Del Castillo configura el joven modelo. Un joven cortés, fiel seguidor y cumplidor de las normas sociales y con especial interés en servir a los demás y a su sociedad, temeroso de Dios y que utiliza con precisión todas las buenas maneras que la urbanidad le da. Esto nos deja un planteamiento final que pone sobre la mesa una cuestión que merece ser reflexionada: ¿Es el manual de Pío Del Castillo opuesto a los valores republicanos que se implementaban en el sistema educacional (Ahern, 1947)? Alarcón, en consonancia con lo que planteara Ahern, asevera:

Con el uso de nuevos textos escolares, fundamentados en la educación laica, los radicales esperaban que desde la escuela los estudiantes adquirieran libertad de conciencia y, por lo tanto, dejaran de lado las actitudes confesionales y los "atavismos religiosos que entorpecían su formación ciudadana"82. Para lograr esto era necesario fortalecer el papel educador e ideológico del Estado para darle a la ciudadanía un contenido eminentemente político, llevar la religión al ámbito privado. (Alarcón, 2012)

¿No están impregnados los Principios de Urbanidad para el Uso de la Juventud de ideas contrarias a esto? Aun cuando es detectable la intención de construcción de nación, ¿verdaderamente refleja este manual una intención de cultivo de libertad de conciencia y formación laica? ¿Deja de lado los "atavismos"? Se hace claro que no. Entonces, ¿Está esta urbanidad planteada por Pío Del Castillo al margen de la intención educacional de la incipiente nación?

Por otra parte, Venegas en su estudio plantea la existencia de una intención distinta:

El objetivo de fondo fue también político, pues además de fortalecer el sistema educativo y refinar las costumbres, los dirigentes garantizaban el statu quo, homogeneizando el comportamiento, siempre asociado a la moral católica, el Gobierno busca asegurarse del respeto hacia las instituciones y la legitimación de su poder político. (Venegas, 2007)

No bastaría el presente ensayo para evaluar en definitiva la intención de la educación y formación moral y ciudadana de Colombia en el siglo XIX, lo que refleja cómo se plantea este como un campo aún por explorar e interpretar a nivel histórico; esta investigación aún no se agota. 


\section{BIBLIOGRAFÍA}

Ahern, E. (1947) El desarrollo de la Educación en Colombia 1820 - 1850. Universidad de California. Berkeley.

Alarcón Meneses, L (2012) Analfabetos pero republicanos. El mundo del libro escolar en el Caribe colombiano, 1857-1886. Historia y Sociedad. Medellín.

Bethell, L. (1986) Historia de América Latina. Editorial Crítica. Barcelona.

Cuervo, R. (1826) Lecciones de Urbanidad. Biblioteca Luis Ángel Arango. Bogotá.

Jaramillo Uribe, J (1964) El pensamiento colombiano en el siglo XIX. Editorial Temis. Bogotá.

Madiedo, M (1863) La ciencia social. Edición Banco de la República. Bogotá.

Venegas, C. (2007) Estudio analítico de manuales de urbanidad colombianos del siglo XIX. Universidad Nacional. Bogotá.

Geraldine Patricia Chacón Villarroel: Venezolana, estudiante de Estudios Liberales y Derecho de la Universidad Metropolitana. Estudiante de Intercambio en el Instituto de Ciencias Políticas de París. Coordinadora de red de Amnistía Internacional Venezuela. Ganadora de múltiples premios a la excelencia académica. Directora de la publicación periódica de la Universidad Metropolitana y parte de diferentes ONGs en Venezuela y Latinoamérica. 